\title{
1 Endogenous SIRT3 activity is dispensable for normal hearing recovery after noise exposure in young
}

\section{2 adult mice}

3 Short title: SIRT3 not required for hearing after mild noise

5 Sally Patel ${ }^{1}$, Lisa Shah ${ }^{1}$, Natalie Dang ${ }^{1}$, Xiaodong Tan ${ }^{2}$, Anthony Almudevar ${ }^{3}$, and Patricia M. White ${ }^{1 *}$.

6

(1) Department of Neuroscience, Ernest J. Del Monte Institute for Neuroscience, University of Rochester School of Medicine and Dentistry, 601 Elmwood Blvd, Rochester, NY.

(2) Department of Otolaryngology-Head and Neck Surgery, Feinberg School of Medicine, Northwestern University, 303 E. Chicago Ave, Chicago, IL 60611.

(3) Department of Biostatistics and Computational Biology, University of Rochester School of Medicine and Dentistry, 601 Elmwood Blvd, Rochester, NY.

$\left({ }^{*}\right)$ To whom correspondence should be addressed.

I5 Corresponding author: Patricia White. Address: Department of Neuroscience, University of Rochester School 16 of Medicine and Dentistry, 601 Elmwood Ave, Rochester, NY 14642, USA. Phone: 1 (585) 273-2340. Email: 


\section{I8 ABSTRACT}

I9 Occupational noise-induced hearing loss (NIHL) affects millions of people worldwide and presents a large

?0 social and personal burden. Some genetic variants in the mitochondrial oxidative stress response correlate

?1 strongly with susceptibility to NIHL in both humans and mice. Here we test the hypothesis that SIRT3, a

?2 regulator of the mitochondrial oxidative stress response, is required in mice for endogenous recovery of

?3 auditory thresholds after a sub-traumatic noise exposure. We expose homozygous Sirt3-KO mice and their

?4 wild-type littermates to a noise dose that confers a temporary threshold shift, but is not sufficient to

25 permanently reduce cochlear function, compromise cell survival, or damage synaptic structures. We find no

?6 difference in hearing function after recovery from noise exposure between the two genotypes, when measured

?7 by either auditory brainstem response (ABR) or distortion product otoacoustic emissions (DPOAE). We show

?8 that SIRT3-specific immunoreactivity is present in outer hair cells, around the post-synaptic regions of inner

?9 hair cells, and faintly within inner hair cells. Nonetheless, outer hair cells and auditory synapses show no

30 increase in loss after noise exposure in the homozygous Sirt3-KO mouse. These data show that SIRT3-

31 dependent processes are not necessary for endogenous hearing recovery after a single, sub-traumatic noise

32 exposure. They demonstrate the existence of cellular mechanisms of cochlear homeostasis in addition to the

33 mitochondrial oxidative stress response. We also present a novel statistical analysis for identifying differences

34 between peak 1 amplitude progressions in ABR waveforms. 


\section{INTRODUCTION}

Noise-induced hearing loss (NIHL) affects at least 10 million adults in the United States [1], including over a million veterans [2]. Noise-induced auditory dysfunction, including tinnitus and NIHL, is the most common disability among former combat soldiers, costing the Veteran's Administration over a billion dollars annually [2]. NIHL is a form of acquired hearing loss, which can be associated with greater levels of anxiety [3], emotional distress [4], and perceived stigmatization [5], as well as poorer health outcomes [6]. There is no approved biological treatment for NIHL [1], and it can only be prevented by physically avoiding noise exposure.

Recent progress has been made in identifying genetic variants that predispose individuals to NIHL, especially in occupational settings $[7,8]$. Gene variants encoding proteins that modulate oxidative stress are over-represented in these studies (for review, see [9]). More specifically, gene variants that reduce mitochondrial function also enhance susceptibility to acquired hearing loss from noise [10,11], ototoxic drugs [12], and age-related hearing loss $[13,14]$. These facts support the interpretation that proteins promoting mitochondrial function and counteracting oxidative stress also protect the cochlea from noise damage. SIRT3 is a mitochondrial lysine deacetylase [15] that promotes an effective oxidative stress response [16] from mitochondrial enzymes, including Superoxide dismutase 2 (SOD2, [17]). SIRT3 variants are also implicated in human longevity [18]. SIRT3 activation has previously been shown to protect cochlear outer hair cells (OHCs) from aging, where investigators used dietary restriction to activate sirtuins. The positive effect was only observed in wild-type, not homozygous Sirt3-KO mice [19]. Importantly, genetic or pharmaceutical activation of sirtuins was also shown to rescue hearing from traumatic noise damage in a SIRT3-dependent manner [20]. Based on its function and these previous SIRT3 gain-of-function findings, we investigated whether there is a requirement for endogenous SIRT3 in the normal recovery of hearing thresholds in adult mice after a temporary threshold shift (TTS). Since TTS is associated with a recoverable disrupted cellular process rather than hair cell loss [21], the role of SIRT3 for restoring mitochondrial function and hearing recovery in subtraumatic noise could be determined by comparing wild-type and Sirt3-KO mice. These loss-of-function studies also complement the already-published gain-of-function studies. Multiple labs have successfully used this method to identify genes [22] and conditions [23] that modulate susceptibility to noise damage. Noise exposure can eliminate high-frequency auditory synapses [21, 24] in a glutamine-dependent manner [25], and the restoration of auditory synapses was proposed to be the mechanism by which SIRT3 reduced NIHL [20]. 
i3 To evaluate any requirement for endogenous SIRT3 activity in resilience from noise damage, we employed a

34 noise exposure that is $80 \%$ of the energy level needed to induce synaptopathy [21, 24]. It induces small or

i5 negligible permanent ABR threshold shifts [22], and does not cause $\mathrm{OHC}$ death [22]. By exposing

i6 homozygous Sirt3-KO mice and their wild-type littermates to this subtraumatic noise, we sought to uncover a

\$7 role for endogenous SIRT3 activation in preserving these cellular structures and functions. 


\section{MATERIALS AND METHODS}

70 Animal usage. All experiments were performed in compliance with the US Department of Health and Human

Services, and were approved by the University Committee on Animal Resources at the University of Rochester

Medical Center or the IACUC at Northwestern University. Male Sirt3-KO mice (129-Sirt3tm1.1Fwa/J; stock number 012755; The Jackson Laboratory) were bred four times to FVB/nJ females (stock number 001800, The Jackson Laboratory). In other experiments, we have found that four generations is sufficient to confer youthful noise damage sensitivity similar to that of congenic FVB/nJ [22, 24]. Heterozygotes with different parents were bred together to obtain both knockout and wild-type littermates. Both males and females were used. In the first five litters generated, fourteen homozygous Sirt3-KO and thirteen wild-type littermates were generated and then exposed to noise. Four additional litters were generated to produce homozygous Sirt3-KO mice and wildtype littermates, which were not exposed to noise, for histology. Mice were given ample nesting materials and small houses within their home cage.

For genotyping, DNA was obtained from 2-mm tail samples that were digested overnight in Proteinase

$\mathrm{K}$ (IBI Sciences) solution at $65^{\circ} \mathrm{C}$ followed by phenol/chloroform extraction. The KAPA Taq PCR kit (Sigma,

BK1000) was used in conjunction with three primer sequences (wild type, CTT CTG CGG CTC TAT ACA

CAG; common, TGC AAC AAG GCT TTA TCT TCC; mutant, TAC TGA ATA TCA GTG GGA ACG) to identify genotypes.

Noise exposure. Awake two month old mice were exposed to noise limited to the 8-16 kHz octave band at 105 decibels for 30 minutes. Mice were each placed into individual triangular wire mesh cages, $12 \mathrm{~cm} \times 5 \mathrm{~cm} \times 5$ $\mathrm{cm}$, in an asymmetric plywood box with a JBL2250HJ compression speaker and JBL2382A biradial horn mounted on the top. This apparatus was contained within a sound booth. The speaker was driven by a TDT RX6 multifunction processor and dedicated attenuator, and controlled with TDT RPvdsEx sound processing software. The sound level was calibrated with a Qwest sound meter, and the sound level was checked each morning with a calibrated iPhone using the FaberAcoustical SoundMeter app. Mice were exposed to noise between 9 am and 4 pm. 
3 Auditory testing. Mice were tested at 7 weeks of age (pre-test), one day after receiving noise exposure, and

37 again fourteen days later. Mice were exposed to noise at P60. Auditory testing was conducted using a Smart

38 EP Universal Smart Box (Intelligent Hearing Systems) with high-frequency speakers from Tucker Davis

39 Systems. Mice were anesthetized with an intraperitoneal injection of ketamine $(80 \mathrm{mg} / \mathrm{kg})$ in a sterile

)0 acepromazine/saline mixture (3 mg/kg). A 10B+ (high frequency transducer/stimulator) probe was placed at

)1 the opening to the external auditory meatus. Sound production in this system was calibrated with a Qwest

)2 sound meter every six months.

Auditory brainstem response (ABR) stimuli were 5-ms clicks, or 5-ms tone pips presented at 5

$\underline{\text { Antibodies. }}$ The following primary antibodies were used: mouse IgG1 anti-SIRT3 antibody (1:200; Novus

Biologicals; RRID:AB_2818991); goat anti-Oncomodulin antibody (OCM; 1:1000; Santa Cruz;

RRID:AB_2267583), rabbit anti-Myosin7a (MYO7; 1:200; Proteus; RRID:AB_10013626) mouse anti-CTBP2 (aka C-Terminal Binding Protein 2; 1:200; BD Transduction Laboratories; RRID:AB_399431), and mouse antiGRIA2 (aka GluR2/GluA2; 1:2000; Millipore; RRID:AB_2113875). The following secondary antibodies were purchased from Jackson Immuno Research: Donkey Anti-Mouse AF488 (1:500; RRID:AB_2340849), Donkey Anti-Rabbit AF594 (1:500; RRID:AB_2340622), Donkey Anti-Rabbit AF647 (1:200; RRID:AB_2340625), Donkey Anti-Goat AF647 (1:200; RRID:AB_2340438), Goat Anti-Mouse AF594 (IgG1, 1:500; 
RRID:AB_2338885), AF488 Goat Anti-Mouse (IgG2a, 1:500; RRID:AB_2338855). For the images in Figure 1,

an AF568 Goat Anti-Mouse (IgG1, 1:200, Thermo Fisher, RRID: AB_2535766) was used.

Tissue preparation for immunostaining. Cochlear organs were dissected out of freshly euthanized animals.

Their stapes were removed, and a hole was made in their apical tips to allow for adequate fluid exchange.

Tissues were immersed in 4\% paraformaldehyde (PFA) in PBS for at least overnight, and decalcified in $0.1 \mathrm{M}$ EDTA at $4^{\circ} \mathrm{C}$ on a rotating platform for four days. These cochleae were decalcified in $10 \%$ EDTA in 1 X PBS

(diluted from 10X PBS, Invitrogen) for 2-3 days. All whole mount preparations were microdissected into turns as previously described [26, 27], and the tectorial membrane was carefully removed. Cochleae were mapped using the ImageJ plug-in from Massachusetts Eye and Ear Infirmary. $\mathrm{mm}$ coverslips for imaging.

Immunostaining. For anti-SIRT3 staining, cochlear pieces were first soaked in $30 \%$ sucrose on a shaker for 20 minutes, and then transferred to dry ice for 10-15 minutes or until the sucrose was completely frozen. The samples were allowed to thaw at room temperature and washed with 1X PBS 3 times, rinsing for 20 minutes in between on a shaker. After blocking with $5 \%$ serum $+1 \%$ Triton, the primary antibody mix diluted in block was added and incubated overnight at $37^{\circ} \mathrm{C}$. After rinsing 3 times in PBS (10 minutes each), secondary antibody mix (diluted in block and including Hoechst or DAPI at 1:1000) was added and incubated away from light for 2 hours at $37^{\circ} \mathrm{C}$. The pieces were then washed another 3 times with 1 X PBS and mounted on slides.

For post-injury histological characterization, dissected mapped turns were immersed in $30 \%$ sucrose, flash frozen in liquid nitrogen, allowed to thaw, washed in room temperature Dulbecco's PBS (Gibco), and blocked for one hour in 1\% Triton / 5\% donkey serum in PBS. Primary antibody incubations of anti-MYO7, anti-OCM, anti-CTBP2, and anti-GRIA2 were performed at $37^{\circ} \mathrm{C}$ for 20 hours. The tissue was washed in PBS, and secondary antibody incubation was performed at $37^{\circ} \mathrm{C}$ for an additional 2 hours, with both MYO7 and OCM labeled with 647-conjugated antibodies. This whole mount protocol was kindly provided by Leslie Liberman. All tissue was mounted using ProLong Gold (Fisher). Whole mounts were placed between two 50 
j0 Confocal microscopy and image processing for figures. Anti-SIRT3 immunostaining was analyzed at the

¡1 Center for Advanced Microscopy/Nikon Imaging Center at Northwestern University, using a Nikon A1 Laser

;2 Scanning or Nikon W1 Dual CAM Spinning Disk imaging setup. Appropriate excitation and emission settings

;3 were used and fixed for all panels in the same images presented in the paper. Post-injury histological

j4 characterizations were done on an Olympus FV1000 laser scanning confocal microscope at the Center for

j5 Advanced Light Microscopy and Nanoscopy at URMC. ImageJ (NIH) was used to Z-project maximal

j6 brightness in confocal stacks. Photoshop (Adobe) was used to set maximal and background levels of

77 projections for the construction of figures.

For cochleogram analysis, composite images for cochleograms were assembled in Photoshop by pasting each optical section into its own layer, and merging the pieces of the optical sections where hair cells were evident. Alternatively, projections of confocal stacks were used when individual hair cells could be clearly distinguished. Composite or projected images for the mapped regions of each organ were assembled in a single file in Photoshop. 100 micron lengths were pasted onto the images on the row of pillar cells, and OHCs were counted to determine the percent lost. Where the loss of OHCs was great enough (>30\%), an average $\mathrm{OHC}$ count, determined from low-frequency regions of the same cochlea, was used as the denominator [28]. Synaptic components of inner hair cells (IHCs) were imaged with a 100X oil lens at 2X magnification. To obtain 3D images of IHCs, the confocal files were opened in Amira 6.0 (Visualization Science Group), appropriately thresholded, and rotated to better display the IHCs. To count ribbon synapses, we visually quantified red/green synaptic puncta, as indicated by colocalized staining within IHCs on projected confocal stacks. The analyst was blinded to genotype, frequency and condition, and the images were randomized in presentation, by renaming the images according to cards drawn from a fair deck. Synapse numbers were then divided by the number of hair cells in the field to obtain a biological replicate. In previous publications, we have used automated synapse detection methods; however, in some of these preparations the staining background for GRIA2 was too variable for computational approaches that use thresholding. This was determined by comparing the number of synapses obtained through visual inspection to those obtained through automated means [29]. 
77 Statistical analysis. Statistical tests for ABR and DPOAE thresholds, and for DPOAE input-output functions,

'8 were performed in $\mathrm{R}$ using standard functions. The data distribution for cochleograms did not meet the

79 Shapiro test for normality, and was therefore assessed with the Kruskal-Wallis rank sum test. Both functions

30 were also performed in R. To accurately calculate significance levels for amplitude progressions, generalized

31 estimating equations (GEE) were used. These are suitable for the analysis of longitudinal data when

32 population level mean responses suffice to resolve hypotheses. The correlation among responses induced by

33 the repeated measure design were thus modelled by constructing a suitable covariance matrix [30].

34 Generalized linear model conventions were used. Responses were modeled as Poisson variates, with

35 varying scale parameter to account for overdispersion. The exchangeable correlation model was used to

36 account for within-sample correlation. The link function was $g(\mu)=\log (\mu)$ so that $\mu=e^{\eta}$, where $\eta$ is a linear

37 prediction term dependent on threshold $x$ and binary group factor $I$. The null hypothesis of no group effect was

38 modelled as $H_{0}: \eta=p o l y(x, d)$, and the alternative hypothesis was modelled as $H \alpha: \eta=p o l y(x, d)$ * $I$, where

$39 \operatorname{poly}(x, d)$ is an order $d$ polynomial with unknown parameters. For the baseline, 14 day post noise (DPN) and

$30 \mathrm{KO}$ analyses, $d=2$ was used, while for the WT analysis $d=4$ proved to be a significantly better fit. Observed

31 significance levels were obtained both by the $\chi^{2}$ approximation method of geeglm, as well as by a parametric

32 bootstrap method. P-values obtained by both methods were comparable. However, the bootstrap procedure

33 was the more conservative, so these P-values were used (see Table 1). 


\section{RESULTS}

Immunofluorescence for SIRT3 protein reveals its expression pattern in the adult mouse cochlea (Fig. 1). Inner and outer hair cells (IHC \& OHC) were visualized with anti-Myosin7 antibodies (Fig. 1, MYO7, green), and nuclei were revealed with DAPI (Fig. 1, blue). SIRT3 immunoreactivity was evident in OHCs and especially in the positions of nerve root endings and supporting cells surrounding the IHCs (Fig. 1a, red). The latter finding is readily observed in the side view (Fig. 1a, cf. red to green). SIRT3 immunoreactivity was significantly greater than that of age-matched, exposure-matched preparations from homozygous Sirt3-KO mice (Fig. 1b, red), demonstrating antibody specificity. After multiple attempts, endogenous SIRT3 protein in the cochlea was not sufficiently abundant for us to visualize on a western blot (not shown). Messenger RNA levels for sirtuins have been previously described in the adult mouse cochlea, with Sirt3 mRNA significantly expressed in adult mouse $\mathrm{IHC}$ and $\mathrm{OHC}[31]$.

\section{Figure 1. SIRT3 is expressed in sensory cells of the adult mouse cochlea.}

a. Cochlear whole mount preparation from a wild-type adult mouse, immunolabeled with antibodies specific to MYO7 (green) and SIRT3 (red), and co-stained with DAPI (blue) to reveal nuclei. The vertical yellow lines show the positions from which the side views are taken. (a') shows anti-SIRT3 immunoreactivity only. b. Cochlear whole mount preparation from a Sirt3-KO adult mouse, identically labeled and imaged. This control was used to set the background to reveal SIRT3-specific staining.

To evaluate SIRT3's role in hearing recovery, we used a noise dose that confers little permanent damage to awake, two month old, wild-type FVB/nJ mice $[22,24]$. An exposure to an 8-16 kHz octave band at $105 \mathrm{~dB}$ for thirty minutes is sufficient to elevate auditory brainstem response (ABR) thresholds in mice one day after noise exposure, which is fully recovered in two weeks. This exposure reduces high-frequency peak 1 amplitudes two weeks after exposure, but does not significantly eliminate high-frequency synapses [24]. Mice underwent hearing tests at around P50, followed by noise exposure at P60, a second hearing test at P61, and a third hearing test at P74 (Fig. 2a). At P74, mice were euthanized for histological analysis.

Figure 2. SIRT3 loss of function does not impact endogenous hearing recovery from noise-induced temporary threshold shifts. 
a. The time course of experiments is shown. Homozygous Sirt3-KO mice and wild-type littermates are tested for ABR and DPOAE thresholds prior to noise exposure (pre-test). At P60, they are exposed to an 8-16 octave band noise at $105 \mathrm{~dB}$ for 30 minutes (noise), which is sufficient to drive temporary threshold shifts in this strain $[22,24]$. Their hearing thresholds are assessed again at 1 day post noise (1 DPN) and 14 days post noise (14 DPN). ABR and DPOAE are assessed at $8,12,16,24$, and $32 \mathrm{kHz}$ for all panels.

b. Mean ABR thresholds prior to noise exposure for homozygous Sirt3-KO mice ( $n=14$, pink) and wild-type littermates ( $n=13$, black). Mice were approximately P50 when tested. Homozygous Sirt3-KO mice had significantly worse overall hearing ( $\mathrm{p}=0.004$ for genotype, two way ANOVA, $\mathrm{n}=27$ mice total); however, no single frequency was significantly worse $(p=0.053,0.39,0.41,0.53,0.059$ for the five frequencies respectively, two-tailed pairwise t-tests at each frequency with Bonferroni correction, $n=27$ mice total).

c. Mean ABR thresholds at 1 DPN for homozygous Sirt3-KO mice $(n=14$, pink) and wild-type littermates $(n=13$, black). No significant difference was seen between genotypes ( $p=0.35$ for genotype, two-way ANOVA, $n=27$ mice total).

d. Mean ABR thresholds at 14 DPN for homozygous Sirt3-KO mice ( $n=14$, pink) and wild-type littermates ( $n=13$, black). No significant difference was seen between genotypes ( $p=0.85$ for genotype, two way ANOVA, $\mathrm{n}=27$ mice total). Overall ANOVA for all three time points showed no significant difference between genotypes $(\mathrm{p}=0.33$, multi-way ANOVA, $\mathrm{n}=27$ mice total).

e. Mean DPOAE thresholds prior to noise exposure for homozygous Sirt3-KO mice ( $n=14$, pink) and wild-type littermates $(n=13$, black). No significant difference was seen between genotypes $(p=0.43$ for genotype, twoway ANOVA, $\mathrm{n}=27$ mice total).

f. Mean DPOAE thresholds at 1 DPN for homozygous Sirt3-KO mice ( $n=14$, pink) and wild-type littermates $(n=13$, black). No significant difference was seen between genotypes ( $p=0.50$ for genotype, two-way ANOVA, $\mathrm{n}=27$ mice total).

g. Mean DPOAE thresholds at 14 DPN for homozygous Sirt3-KO mice ( $n=14$, pink) and wild-type littermates ( $n=13$, black). No significant difference was seen between genotypes ( $p=0.25$ for genotype, two-way ANOVA, $\mathrm{n}=27$ mice total). Error bars: s.e.m.

We found that prior to noise exposure, homozygous Sirt3-KO mice had slightly but significantly worse ABR thresholds on average compared to wild-type littermates (Fig. 2b, pink traces, $p=0.004$ for genotype, two- 
18 way ANOVA, $n=27$ mice total). Notably, no single frequency was significantly worse $(p=0.053,0.39,0.41$,

0.53, 0.059 for $8,12,16,24$, and $32 \mathrm{kHz}$ frequencies respectively, two-tailed pairwise t-tests at each frequency

with Bonferroni correction, $\mathrm{n}=27$ mice total). At $1 \mathrm{DPN}$, homozygous Sirt3-KO mice had significantly elevated

ABR thresholds (Fig 2c, pink traces, $p=6 \times 10^{-14}$, for time point, two way ANOVA, $n=14$ mice). ABR thresholds were also elevated for wild-type littermates (Fig. 2c, black traces, $p=2 \times 10^{-16}$ for time point, two-way ANOVA, $\mathrm{n}=13$ mice). Thresholds for homozygous Sirt3-KO and wild-type mice were not different at 1 DPN (Fig. 2c, $\mathrm{p}=0.35$ for genotype at $1 \mathrm{DPN}$, two-way ANOVA, $n=27$ mice). At $14 \mathrm{DPN}$, there was also no difference in ABR

calculated thresholds for distortion product otoacoustic emissions (DPOAE) for the same mice at the same time points (Fig. 2e-g). DPOAE thresholds for both genotypes were similarly elevated at 1 DPN for both genotypes, and recovered to the same levels at 14 DPN ( $p=0.13$ for genotype, multivariate ANOVA, $n=27$ mice).

We evaluated the requirement for SIRT3 on ABR peak 1 amplitudes of homozygous Sirt3-KO mice and

their wild-type littermates before and after noise damage. Mean ABR thresholds for homozygous Sirt3-KO mice were $55.7 \mathrm{~dB} \pm 4.1$ at $32 \mathrm{kHz}$, compared to $44.2 \pm 4.1$ for wild-type littermates, which was not different when adjusted for multiple comparisons. Note that thresholds are determined by the continued presence of any part of the waveform, and not solely by the presence of peak 1 . To assess differences between peak 1 amplitude progressions, we used generalized estimating equations to build linear models of the correlated data, as these are suited for the analysis of longitudinal data when population level mean responses suffice to resolve hypotheses [30]. Fig. 3 shows pairwise comparisons of data from each mouse (Fig. 3a-d, light lines), the linear models (Fig. 3a-d, heavy lines) as well as mean latency values at both time points for peak 1 (Fig. e, f). These values of the amplitude integrals and their associated p-values are listed in Table 1 (see Methods for a full description). We propose that the amplitude integrals (Table 1) are appropriate for evaluating differential recruitment of auditory synapses as the stimulus is amplified, when genotypes and time points are compared. Mean peak 1 amplitudes were significantly reduced for homozygous Sirt3-KO mice compared to wild-types at $32 \mathrm{kHz}$, after adjusting for multiple comparison using this approach $(\mathrm{p}=0.046$, parametric bootstrap method, $\mathrm{n}=27$ mice). Any difference between the two datasets was lost at 14 DPN, as mean peak 1 amplitudes at 32 $\mathrm{kHz}$ were significantly reduced in wild-type littermates $(\mathrm{p}=0.002$, parametric bootstrap method, $n=13$ mice) to 
76 similar levels as homozygous Sirt3-KO mice ( $\mathrm{p}=0.87$, parametric bootstrap method, $\mathrm{n}=27$ mice). Curiously,

77 mean peak 1 amplitudes at $32 \mathrm{kHz}$ in homozygous Sirt3-KO mice were unchanged between conditions

$78(p=0.914$, parametric bootstrap method, $n=14)$. Latencies for peak 1 were not different between genotypes at

79 either time point (Fig. 3e, f, cf. black to red).

\section{0 \\ Figure 3. Sirt3 loss of function impacts pre-noise ABR peak 1 amplitudes, but not amplitudes after}

noise exposure.

a. Peak 1 amplitudes in ABR measurements for $32 \mathrm{kHz}$ stimuli prior to noise exposure for homozygous Sirt3-

$K O$ mice $(n=14$, thin red) and wild-type littermates ( $n=13$, thin black). Peak 1 amplitudes (microvolts) are

plotted on the $y$ axis for different amplitudes of stimulus (dB SPL) plotted on the $x$-axis. Heavier red and black

lines represent respective GEE models. The area under the curve, representative of the progressive neuronal

recruitment, is significantly reduced for homozygous Sirt3-KO mice ( $p=0.046$, parametric bootstrap method,

$\mathrm{n}=27$ mice).

b. Same analysis as in (a), but for peak 1 amplitude values obtained from the same mice 14 DPN. No difference is seen between genotypes ( $p=0.87$, parametric bootstrap method, $n=27$ mice).

c. Same analysis as in $(a, b)$, however, in this case amplitudes obtained from homozygous Sirt3-KO mice are compared before and after noise exposure. No differences are seen ( $p=0.914$, parametric bootstrap method, $n=14)$.

c. Same analysis as in (a, b), however, in this case amplitudes obtained from wild-type littermates are compared before and after noise exposure. A significant reduction in amplitude is evident after noise exposure (cf. red to black, $p=0.002$, parametric bootstrap method, $n=13$ ).

e. Mean peak 1 latencies for $32 \mathrm{kHz}$ stimuli prior to noise exposure for homozygous Sirt3-KO mice $(n=14$, pink) and wild-type littermates ( $n=13$, black). No differences are seen between genotypes.

f. Mean peak 1 latencies for $32 \mathrm{kHz}$ stimuli at 14 DPN for homozygous Sirt3-KO mice ( $\mathrm{n}=14$, pink) and wildtype littermates ( $n=13$, black). No differences are seen between genotypes. Error bars: s.e.m.

Table 1. Integrals and p-values for $32 \mathrm{kHz}$ ABR voltage progressions for homozygous Sirt3-KO and wild-type mice. 


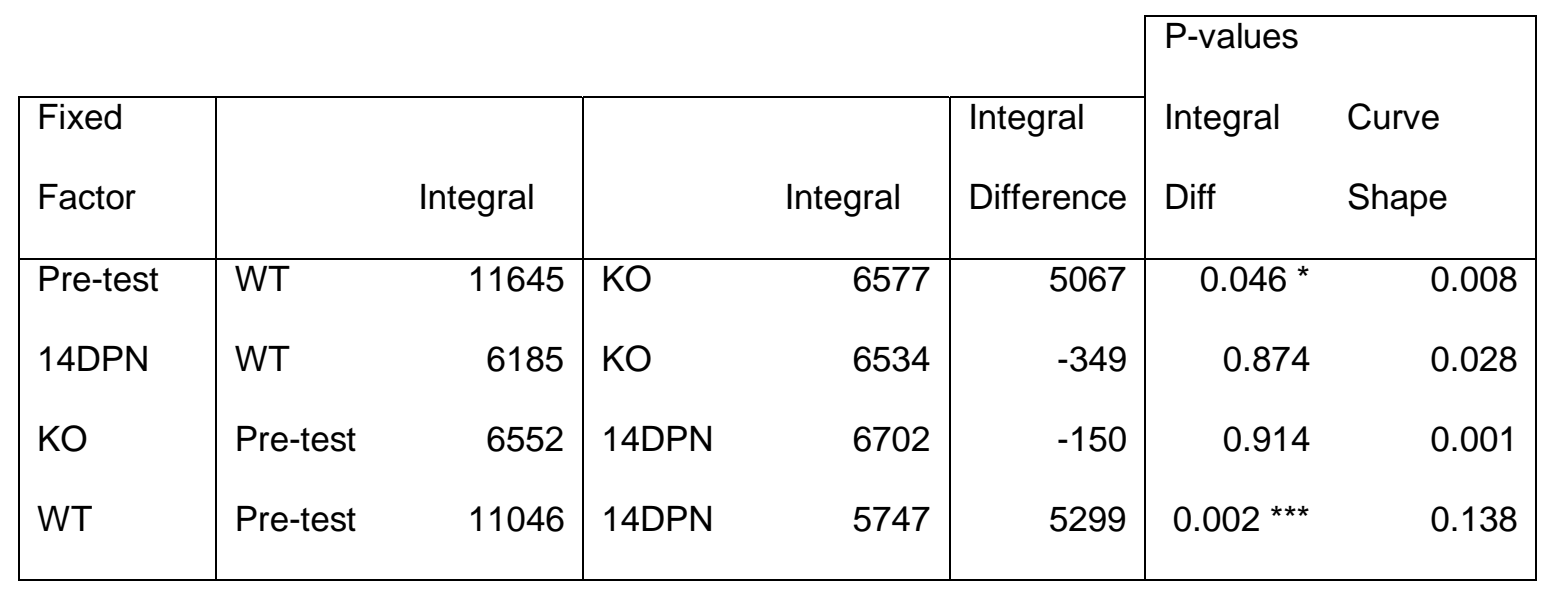

We evaluated the requirement for SIRT3 on DPOAE fine structure by comparing input-output functions for homozygous Sirt3-KO and wild-type mice, prior to noise exposure and at 14 DPN (Fig. 4). Values for each

)4 L2 frequency are displayed separately, comparing homozygous Sirt3-KO mice (Fig. 4, pink) to wild-type

)5 littermates (Fig. 4, black). The curves overlapped at each frequency, and no significant differences were )6 observed ( $p=0.07$ for genotype, multivariate ANOVA, $n=27$ mice). Taken together, these data suggest that the absence of endogenous SIRT3 activity does not confer susceptibility to permanent functional impairment from noise exposure, by these measures.

Figure 4. No differences in input-output functions for DPOAE between homozygous Sirt3-KO mice and wild-type littermates at any time point.

Ten graphs are arranged by time point, with pre-test results on the top row and 14 DPN results on the bottom row, as well as by frequency, displaying $8 \mathrm{kHz}, 12 \mathrm{kHz}, 16 \mathrm{kHz}, 24 \mathrm{kHz}$, and $32 \mathrm{kHz}$ results from left to right. Mean distortion product (DP) amplitudes in $\mathrm{dB}$ are plotted on the $\mathrm{y}$-axis, and the amplitudes of the L2 stimulus $(\mathrm{dB})$ are plotted on the $y$-axis. No differences between the genotypes ( $p=0.07$ for genotype, multivariate ANOVA, $\mathrm{n}=27$ mice). Error bars: s.e.m.

Given SIRT3's immunoreactivity in OHCs (Fig. 1), we sought to determine if these cells are more susceptible to cell death from noise in the absence of SIRT3. We prepared mapped cochlear organs for whole mount imaging of OHCs and IHCs for cochleogram analysis. The cochleae were divided into 100 micron segments along the line of pillar cells, surviving $\mathrm{OHCs}$ were quantified for each segment, and the percent of lost $\mathrm{OHCs}$ plotted as a function of distance along the cochlear length (Fig. 5). Basal OHCs were more prone to losses in both the wild-type and homozygous Sirt3-KO mouse in the absence of noise damage (Fig. 5, cf. b to 
?2 f), with some variability between biological replicates (Fig. 5b, cf. purple and green). Strikingly, noise exposure

?3 did not potentiate OHC losses in the homozygous Sirt3-KO mouse (Fig. 5, cf. h to f, p=0.49, Kruskal-Wallis

?4 rank sum test, $\mathrm{n}=3-4$ biological replicates). No changes in $\mathrm{IHC}$ survival were observed for either genotype in

?5 either condition (data not shown). These data indicate that SIRT3 loss of function does not significantly impact

?6 OHC survival after mild noise insult.

Figure 5. No apparent differences in OHC survival for homozygous Sirt3-KO mice and wild-type

littermates prior to noise exposure or at 14 DPN.

a. Representative cochlear preparation from a wild-type littermate without noise exposure, with IHCs revealed

with anti MYO7 antibodies (white) and OHCs revealed with anti-OCM antibodies (also white). Scale bar: 200

microns.

b. Cochleogram results from 4 mapped cochlear preparations from 4 wild-type littermates without noise exposure, where $\mathrm{OHC}$ loss was quantified in 100 micron segments. The distance from the apex for each segment is plotted on the $\mathrm{x}$-axis, and the percent $\mathrm{OHC}$ loss for the segment is plotted on the $y$-axis. Dots with the same color (purple, blue, green, or red) are from the same cochlear preparation.

c. Representative cochlear preparation from a wild-type littermate at 14 DPN, with the same staining as in (a). d. Cochleogram results, similar to (b), from 3 mapped cochlear preparations from 3 wild-type littermates at 14 DPN. Results are similar to (b).

e. Representative cochlear preparation from a homozygous Sirt3-KO mouse without noise exposure, with the same staining as in (a).

f. Cochleogram results, similar to (b), from 4 mapped cochlear preparations from 4 homozygous Sirt3-KO mice without noise exposure. Results are similar to (b).

g. Representative cochlear preparation from a homozygous Sirt3-KO mouse at 14 DPN, with the same staining as in (a).

h. Cochleogram results, similar to (b), from 4 mapped cochlear preparations from 4 homozygous Sirt3-KO mice at 14 DPN. Results are similar to $(f)$ and (b). 
noise exposure in the homozygous Sirt3-KO mouse. We imaged MYO7+ IHCs at 12 and $24 \mathrm{kHz}$ that had also

been stained with antibodies against CTBP2, the major component of auditory synaptic ribbons, as well as

GRIA2, one of the glutamate receptor components of auditory post-synaptic specializations [32].

Representative images for wild-type and homozygous Sirt3-KO cochleae, with and without noise exposure, are depicted in Fig. 6. Confocal stacks were imported into Amira, so that the IHCs could be rotated, to view at similar angles. Paired synaptic structures were quantified visually on randomized projected images, whose condition and genotype were masked from the analyst. Each frequency, condition, and genotype is labeled with the mean number of synapses per IHC for 3-4 biological replicates. Wild-type cochlea harbored between 16.7 and 18.0 synapses per IHC in no noise condition (Fig. 6a, b). These numbers were not significantly reduced after noise exposure (Fig. 6c, d), similar to previous results [22, 24]. At both frequencies and in both conditions, homozygous Sirt3-KO cochleae harbored between 17.8 and 18.8 synapses per IHC (Fig. 6i-I). Thus, we find no evidence that the loss of SIRT3 protein potentiates synaptopathy from noise exposure.

\section{Figure 6. Synaptic loss is not potentiated in homozygous Sirt3-KO mice after noise exposure.}

a. Representative image of wild-type $12 \mathrm{kHz}$ IHCs with no noise exposure, stained with anti-MYO7 (blue), antiCTBP2 (red) to reveal pre-synaptic structures, and anti-GRIA2 (green) to reveal post-synaptic structures.

Mean synaptic number per IHC ( \pm s.e.m.), visually counted from 3 biological replicates with $8 \mathrm{IHCs}$ each, is noted in the upper right hand corner. Size bar: 5 microns.

b. Representative image of wild-type $24 \mathrm{kHz} \mathrm{IHCs}$ with no noise exposure, similarly stained to (a). 3 replicates, 6-7 IHCs each.

c. Representative image of wild-type $12 \mathrm{kHz}$ IHCs at $14 \mathrm{DPN}$, similarly stained to (a). 4 replicates, 7-11 IHCs each.

d. Representative image of wild-type $24 \mathrm{kHz}$ IHCs at 14 DPN, similarly stained to (a). 3 replicates, 7-8 IHCs each.

e $-\mathrm{h}$. Three dimensional Amira rendering of confocal stacks shown in $(a-d)$, respectively, with MYO7 rendered in white, CTBP2 in red, and GRIA2 in green. Hair cells are rotated to display from similar orientations. 
75 i. Representative image of homozygous Sirt3-KO $12 \mathrm{kHz}$ IHCs with no noise exposure, similarly stained to (a).

763 replicates, 6-8 IHCs each.

77 j. Representative image of homozygous Sirt3-KO $24 \mathrm{kHz}$ IHCs with no noise exposure, similarly stained to (a).

783 replicates, 4-7 IHCs each.

79 k. Representative image of homozygous Sirt3-KO $12 \mathrm{kHz}$ IHCs at 14 DPN, similarly stained to (a). The angle

30 of the tissue in the imaging foreshortens the IHC. 5 replicates, 6-8 IHCs each.

31 I. Representative image of homozygous Sirt3-KO $24 \mathrm{kHz}$ IHCs at 14 DPN, similarly stained to (a). IHCs

32 appear different because of the angle of the tissue. 3 replicates, 6-8 IHCs each.

$33 \mathrm{~m}$ - p. Three-dimensional Amira rendering of confocal stacks shown in $(\mathrm{i}-\mathrm{I})$, respectively, with MYO7

34 rendered in white, CTBP2 in red, and GRIA2 in green. Hair cells are rotated to display from similar

35 orientations. The apical truncation shown in $(p)$ is an artifact of the confocal imaging. 


\section{DISCUSSION}

NIHL is a life-long, progressive disability that presents a significant social and personal burden.

Variants in genes that reduce oxidative stress can enhance susceptibility to occupational NIHL [7], indicating the importance of this pathway to acquired hearing disorders. SIRT3 is a major regulator of the mitochondrial oxidative stress response [33]. We tested the hypothesis that loss of SIRT3 function could impact endogenous recovery from a mild noise insult in young adult mice. We show that SIRT3 immunoreactivity is present in OHCs, around the post-synaptic structures of IHCs, and faintly within IHCs themselves, comparable to mRNA studies [31]. We measured auditory thresholds of homozygous Sirt3-KO mice and their wild-type littermates with $\mathrm{ABR}$ and DPOAE, before noise exposure, immediately after noise exposure, and a third time after the mice had recovered. No differences were observed between the genotypes after noise exposure. We did not observe permanent adverse effects on homozygous Sirt3-KO OHCs, either by DPOAE fine structure analysis or in their survival after noise damage. We also did not observe any potentiation of high-frequency synaptic loss in homozygous Sirt3-KO mice. We did observe a slight but significant reduction in peak 1 amplitude at 32 $\mathrm{kHz}$ for homozygous Sirt3-KO mice, but this effect was evident prior to noise damage and was not further potentiated. In this finding, we present a new statistical analysis for peak 1 comparisons. Taken together, these results strongly indicate that SIRT3 is not required in mice for endogenous recovery from a single noise exposure.

The oxidative stress response acts to mitigate the effects of traumatic noise injury on the cochlea [11]. Mitochondrial genetic defects are strongly associated with NIHL susceptibility [10], and strategies to reduce mitochondrial oxidative stress can protect from NIHL in animal models [34, 35]. SIRT3 protects by activating mitochondrial enzymes that reduce oxidative radicals, including SOD2 [36] and Catalase [37], through lysine deacetylation. Notably, the exogenous activation of SIRT3 through pharmacological means restores partial cochlear function after traumatic noise damage [20] and aminoglycoside damage [38]. In analyses of SIRT3 effector molecules, heterozygous Sod2-KO mice were shown to incur more damage from traumatic noise compared to wild-type controls, including higher ABR thresholds and greater OHC losses [39]. Together, these published data show that in mice, the mitochondrial oxidative stress response is activated after traumatic noise, reduced levels of those effectors worsen trauma outcomes, and increasing the stress response through 
I3 greater levels of SIRT3 activity improves trauma outcomes. This is consistent with current models of human

I4 occupational NIHL susceptibility, and informs some efforts to combat NIHL [40, 41].

I5 In contrast, the experiments in this report assess endogenous recovery after a single sub-traumatic

16 noise exposure. We have previously shown that this paradigm reveals susceptibility to noise damage in

17 homozygous Foxo3-KO mice [22]. Others have used similar paradigms to investigate the protective roles of

I 8 the medial olivocochlear system [42] and of peroxisomes [43]. In all three examples, a sub-traumatic noise

I9 exposure readily revealed a genetic susceptibility to damage. Given SIRT3's expression in OHCs (Fig. 1), its

?0 role in activating the oxidative stress response, and the sheer importance of that response in mitigating

?1 traumatic noise damage, we were surprised to find no sub-traumatic susceptibility with SIRT3 loss-of-function

?2 mice (Figs. 2, 4, \& 5). These data may be interpreted to indicate that for a single, sub-traumatic noise

exposure, some other cellular protective mechanism is sufficient for endogenous recovery. Heat shock

proteins [44] and the FOXO3-dependent response [22] are candidates. Alternatively, a clue may lie in the

expression pattern of SIRT3: it may be that the oxidative stress response in SIRT3-negative cells, including

supporting cells and/or the stria vascularis, is sufficient to protect OHCs from sub-traumatic noise. We

emphasize that these results indicate that cochlear responses to chronic insults, such as aging and

occupational noise exposure, contain different or additional components, when compared to cochlear

responses to a single, acute insult. In fact, a profound relationship between TTS and age-related hearing loss

has been reported, in which acute synaptopathy was associated with an exacerbated long-term change of cochlear function [21]. In this regard, whether the presence of SIRT3 makes a difference in the situation of cochlear synaptopathy or in long-term changes after sub-traumatic noise exposure is worth further investigation.

We also show that high-frequency cochlear synaptopathy from noise exposure is not potentiated in the homozygous Sirt3-KO mouse (Fig. 6). This result was also counterintuitive, given the prominent role of excitotoxic glutamate in auditory synaptic loss [21, 45-47] and the presence of SIRT3 immunoreactivity around the synaptic region of the IHCs (Fig. 1). The latter finding suggests that SIRT3 may promote mitochondrial function in neurites and supporting cells, and would be predicted to be protective. However, we note that the homozygous Sirt3-KO mouse would have had mitochondrial dysfunction throughout its development. It is possible that compensatory mechanisms to prevent calcium overload are present in homozygous Sirt3-KO 
neurites. Such mechanisms could take the form of changes in efferent neurotransmission. Exogenous dopamine, for example, can lower cochlear action potential amplitudes in guinea pigs, providing a tonic effect that counteracts excitotoxicity [48]. Alternatively, stress signaling within spiral ganglion afferent neurites could modulate their activity. Altered cAMP levels within spiral ganglion neurites, for example, modulate an inward potassium current through HCN channels, which shapes EPSP waveforms and modulates the probability of spike formation [49]. Other explanations are also possible.

The amplitude progressions of ABR peak 1 represent the only significant difference observed between homozygous Sirt3-KO mice and their wild-type littermates prior to noise exposure (Fig. 3). We present the novel use of an advanced statistical method, generalized estimating equations, to more accurately calculate significant differences between amplitude progressions [30]. While the amplitude progression is sometimes modeled as a straight line, we contend that this is an unnecessary oversimplification, given the newly described molecular diversity of the responding spiral ganglion population [50]. Generalized estimating equations can provide a function that is a more accurate representation of the progression. Any increases seen in the amplitude progression reflect additional spiral ganglion neurons participating in the synchronized response, as the tone pip gets louder. Since the summation of neuronal activity is the variable of interest, we contend that the integral of the amplitude progression function is the appropriate measure for comparison. Our analysis contrasts with comparing the slopes of two progressions, as these are the derivatives of the neuronal summation. Of course, this method is only valid when thresholds are not statistically different, as we had found for the $32 \mathrm{kHz}$ frequency (Fig. 2). It is also important to point out that the individual scoring the threshold must score on the whole waveform and not solely on the presence or absence of peak 1.

Compensatory mechanisms could also account for the reduced peak 1 amplitude observed in homozygous Sirt3-KO mice prior to noise exposure (Fig. 3). Note that in the wild-type mice, peak 1 amplitudes are significantly reduced in response to noise, as we have previously reported [24]. These differences in peak 1 amplitude are not due to synaptopathy, as the numbers of synapses are not different between the genotypes or between conditions. Instead, it must be due to either reduced or desynchronized firing by spiral ganglion neurons. These findings underscore the adaptive nature of the adult cochlea in its response to stimuli in the context of genomic variability, and indicate that cellular stress responses additional to the oxidative stress response promote cochlear homeostasis for sub-traumatic challenges. 


\section{i9 ACKNOWLEDGEMENTS}

70 This work was supported by the NIDCD with R01 DC014261, awarded to PMW, and in part by a grant from the

71 American Hearing Research Foundation, awarded to XT. The confocal imaging for SIRT3 expression was

72 performed at the Northwestern University Center for Advanced Microscopy, generously supported by NCI

73 CCSG P30 CA060553 awarded to the Robert H Lurie Comprehensive Cancer Center. We gratefully

74 acknowledge Dr. Anne Luebke, who maintains the Small Animal Auditory Testing Core and Dr. V. Kaye

75 Thomas, who maintains the Center for Advanced Light Microscopy and Nanoscopy, both at URMC. We also

76 thank Ms. Holly Beaulac for a critical reading of the manuscript.

77 SP, XT, and PMW planned experiments; SP, LS, ND, and XT performed experiments; LS, ND, XT, AA,

78 and PMW analyzed data; PMW wrote the manuscript draft; and XT, AA, and PMW edited the manuscript. The

79 authors have no competing interests. 
1. NIDCD. Noise Induced Hearing Loss Bethesda, MD: National Institutes of Health; 2019 [updated May 31, 2019; cited 2019]. Available from: https://www.nidcd.nih.gov/health/noise-induced-hearing-loss.

2. Annual-Benefit-Report. Annual Benefit Report FY 2014. In: Administration V, editor.: Dept. of Veterans Affairs Veterans Benefits Administration; 2014.

3. Ferrari S, Monzani D, Gherpelli C, MacKinnon A, Mongelli F, Federici G, et al. Acquired Hearing Loss, Anger, and Emotional Distress: The Mediating Role of Perceived Disability. J Nerv Ment Dis. 2019;207(6):45966. doi: 10.1097/NMD.0000000000000995. PubMed PMID: 31058747.

4. Thomas AJ. Acquired deafness and mental health. Br J Med Psychol. 1981;54(Pt 3):219-29. doi: 10.1111/j.2044-8341.1981.tb01452.x. PubMed PMID: 7272249.

5. Southall K, Gagne JP, Jennings MB. Stigma: a negative and a positive influence on help-seeking for adults with acquired hearing loss. International journal of audiology. 2010;49(11):804-14. doi: 10.3109/14992027.2010.498447. PubMed PMID: 20831460.

6. Gopinath B, Schneider J, Hickson L, McMahon CM, Burlutsky G, Leeder SR, et al. Hearing handicap, rather than measured hearing impairment, predicts poorer quality of life over 10 years in older adults. Maturitas. 2012;72(2):146-51. doi: 10.1016/j.maturitas.2012.03.010. PubMed PMID: 22521684.

7. White P. Genetic susceptibility to hearing loss from noise. The Hearing Journal. 2019;In Press.

8. Sliwinska-Kowalska M, Pawelczyk M. Contribution of genetic factors to noise-induced hearing loss: a human studies review. Mutation research. 2013;752(1):61-5. doi: 10.1016/j.mrrev.2012.11.001. PubMed PMID: 23207014.

9. Clifford RE, Hoffer M, Rogers R. The Genomic Basis of Noise-induced Hearing Loss: A Literature Review Organized by Cellular Pathways. Otol Neurotol. 2016;37(8):e309-16. doi: 10.1097/MAO.0000000000001073. PubMed PMID: 27518140.

10. Yu J, Wang Y, Liu P, Li Q, Sun Y, Kong W. Mitochondrial DNA common deletion increases susceptibility to noise-induced hearing loss in a mimetic aging rat model. Biochem Biophys Res Commun. 2014;453(3):515-20. doi: 10.1016/j.bbrc.2014.09.118. PubMed PMID: 25285633.

11. Bottger EC, Schacht J. The mitochondrion: a perpetrator of acquired hearing loss. Hear Res. 2013;303:12-9. doi: 10.1016/j.heares.2013.01.006. PubMed PMID: 23361190; PubMed Central PMCID: PMCPMC3681877.

12. Yuan H, Qian Y, Xu Y, Cao J, Bai L, Shen W, et al. Cosegregation of the G7444A mutation in the mitochondrial COI/tRNA(Ser(UCN)) genes with the 12S rRNA A1555G mutation in a Chinese family with aminoglycoside-induced and nonsyndromic hearing loss. Am J Med Genet A. 2005;138A(2):133-40. doi: 10.1002/ajmg.a.30952. PubMed PMID: 16152638; PubMed Central PMCID: PMCPMC2759106.

13. Kujoth GC, Hiona A, Pugh TD, Someya S, Panzer K, Wohlgemuth SE, et al. Mitochondrial DNA mutations, oxidative stress, and apoptosis in mammalian aging. Science. 2005;309(5733):481-4. doi: 10.1126/science.1112125. PubMed PMID: 16020738.

14. Markaryan A, Nelson EG, Hinojosa R. Quantification of the mitochondrial DNA common deletion in presbycusis. Laryngoscope. 2009;119(6):1184-9. doi: 10.1002/lary.20218. PubMed PMID: 19358252.

15. Lombard DB, Alt FW, Cheng HL, Bunkenborg J, Streeper RS, Mostoslavsky R, et al. Mammalian Sir2 homolog SIRT3 regulates global mitochondrial lysine acetylation. Mol Cell Biol. 2007;27(24):8807-14. doi: 10.1128/MCB.01636-07. PubMed PMID: 17923681; PubMed Central PMCID: PMC2169418.

16. Kim HS, Patel K, Muldoon-Jacobs K, Bisht KS, Aykin-Burns N, Pennington JD, et al. SIRT3 is a mitochondria-localized tumor suppressor required for maintenance of mitochondrial integrity and metabolism during stress. Cancer Cell. 2010;17(1):41-52. doi: 10.1016/j.ccr.2009.11.023. PubMed PMID: 20129246; PubMed Central PMCID: PMC3711519.

17. Qiu X, Brown K, Hirschey MD, Verdin E, Chen D. Calorie restriction reduces oxidative stress by SIRT3mediated SOD2 activation. Cell metabolism. 2010;12(6):662-7. doi: 10.1016/j.cmet.2010.11.015. PubMed PMID: 21109198.

18. Bellizzi D, Rose G, Cavalcante P, Covello G, Dato S, De Rango F, et al. A novel VNTR enhancer within the SIRT3 gene, a human homologue of SIR2, is associated with survival at oldest ages. Genomics. 2005;85(2):258-63. doi: 10.1016/j.ygeno.2004.11.003. PubMed PMID: 15676284.

19. Someya S, Yu W, Hallows WC, Xu J, Vann JM, Leeuwenburgh C, et al. Sirt3 mediates reduction of oxidative damage and prevention of age-related hearing loss under caloric restriction. Cell. 2010;143(5):80212. doi: 10.1016/j.cell.2010.10.002. PubMed PMID: 21094524; PubMed Central PMCID: PMC3018849. 
bioRxiv preprint doi: https://doi.org/10.1101/2020.02.05.935882; this version posted February 10, 2020. The copyright holder for this preprint (which was not certified by peer review) is the author/funder, who has granted bioRxiv a license to display the preprint in perpetuity. It is made available under aCC-BY-NC-ND 4.0 International license.

20. Brown KD, Maqsood S, Huang JY, Pan Y, Harkcom W, Li W, et al. Activation of SIRT3 by the NAD Precursor Nicotinamide Riboside Protects from Noise-Induced Hearing Loss. Cell metabolism. 2014;20(6):1059-68. doi: 10.1016/j.cmet.2014.11.003. PubMed PMID: 25470550.

21. Kujawa SG, Liberman MC. Adding Insult to Injury: Cochlear Nerve Degeneration after "Temporary" Noise-Induced Hearing Loss. J Neurosci. 2009;29:14077-85.

22. Gilels F, Paquette ST, Beaulac HJ, Bullen A, White PM. Severe hearing loss and outer hair cell death in homozygous Foxo3 knockout mice after moderate noise exposure. Scientific reports. 2017;7(1):1054. doi: 10.1038/s41598-017-01142-3. PubMed PMID: 28432353; PubMed Central PMCID: PMC5430619.

23. Cederroth CR, Park JS, Basinou V, Weger BD, Tserga E, Sarlus H, et al. Circadian Regulation of Cochlear Sensitivity to Noise by Circulating Glucocorticoids. Current biology : CB. 2019;29(15):2477-87 e6. doi: 10.1016/j.cub.2019.06.057. PubMed PMID: 31353184.

24. Paquette ST, Gilels F, White PM. Noise exposure modulates cochlear inner hair cell ribbon volumes, correlating with changes in auditory measures in the FVB/nJ mouse. Scientific reports. 2016;6:25056. doi: 10.1038/srep25056. PubMed PMID: 27162161; PubMed Central PMCID: PMC4861931.

25. Kim KX, Payne S, Yang-Hood A, Li SZ, Davis B, Carlquist J, et al. Vesicular Glutamatergic Transmission in Noise-Induced Loss and Repair of Cochlear Ribbon Synapses. J Neurosci. 2019;39(23):443447. doi: 10.1523/JNEUROSCI.2228-18.2019. PubMed PMID: 30926748; PubMed Central PMCID: PMCPMC6554621.

26. Liberman MC. Video Tutorial for Cochlear Dissection Boston, MA: Massachusetts Eye and Ear; 2009 [updated 02/05/09; cited 2012]. Available from: http://www.masseyeandear.org/research/otolaryngology/investigators/laboratories/eaton-peabodylaboratories/epl-histology-resources/video-tutorial-for-cochlear-dissection.

27. Montgomery SC, Cox BC. Whole Mount Dissection and Immunofluorescence of the Adult Mouse Cochlea. Journal of visualized experiments : JoVE. 2016;(107). doi: 10.3791/53561. PubMed PMID: 26779585. 28. Viberg A, Canlon B. The guide to plotting a cochleogram. Hear Res. 2004;197(1-2):1-10. doi: 10.1016/j.heares.2004.04.016. PubMed PMID: 15504598.

29. Gilels F, Paquette ST, Zhang J, Rahman I, White PM. Mutation of Foxo3 causes adult onset auditory neuropathy and alters cochlear synapse architecture in mice. J Neurosci. 2013;33(47):18409-24.

30. McCulloch CE, Searle SR, Neuhas JM. Generalized, Linear, and Mixed Models Hoboken, NJ: WileyInterscience; 2008.

31. Hertzano R, Orvis J. gEAR - gene Expression Analysis Resource web portal: University of Maryland; 2016 [cited 2017]. Available from: http://gear.igs.umaryland.edu/index.html.

32. Liberman LD, Wang H, Liberman MC. Opposing gradients of ribbon size and AMPA receptor expression underlie sensitivity differences among cochlear-nerve/hair-cell synapses. J Neurosci. 2011;31(3):801-8. doi: 10.1523/JNEUROSCI.3389-10.2011. PubMed PMID: 21248103; PubMed Central PMCID: PMC3290333.

33. Giblin W, Skinner ME, Lombard DB. Sirtuins: guardians of mammalian healthspan. Trends in genetics : TIG. 2014;30(7):271-86. doi: 10.1016/j.tig.2014.04.007. PubMed PMID: 24877878; PubMed Central PMCID: PMC4077918.

34. Fetoni AR, De Bartolo P, Eramo SL, Rolesi R, Paciello F, Bergamini C, et al. Noise-induced hearing loss ( $\mathrm{NIHL}$ ) as a target of oxidative stress-mediated damage: cochlear and cortical responses after an increase in antioxidant defense. J Neurosci. 2013;33(9):4011-23. doi: 10.1523/JNEUROSCI.2282-12.2013. PubMed PMID: 23447610; PubMed Central PMCID: PMCPMC6619303.

35. Henderson D, Bielefeld EC, Harris $\mathrm{KC}, \mathrm{Hu} \mathrm{BH}$. The role of oxidative stress in noise-induced hearing loss. Ear Hear. 2006;27(1):1-19. Epub 2006/02/01. doi: 10.1097/01.aud.0000191942.36672.f3 00003446-200602000-00001 [pii]. PubMed PMID: 16446561.

36. Tao R, Coleman MC, Pennington JD, Ozden O, Park SH, Jiang H, et al. Sirt3-mediated deacetylation of evolutionarily conserved lysine 122 regulates MnSOD activity in response to stress. Mol Cell. 2010;40(6):893904. doi: 10.1016/j.molcel.2010.12.013. PubMed PMID: 21172655; PubMed Central PMCID: PMCPMC3266626.

37. Sundaresan NR, Gupta M, Kim G, Rajamohan SB, Isbatan A, Gupta MP. Sirt3 blocks the cardiac hypertrophic response by augmenting Foxo3a-dependent antioxidant defense mechanisms in mice. J Clin Invest. 2009;119(9):2758-71. doi: 10.1172/JCI39162. PubMed PMID: 19652361; PubMed Central PMCID: PMC2735933. 
38. Quan Y, Xia L, Shao J, Yin S, Cheng CY, Xia W, et al. Adjudin protects rodent cochlear hair cells against gentamicin ototoxicity via the SIRT3-ROS pathway. Scientific reports. 2015;5:8181. doi:

10.1038/srep08181. PubMed PMID: 25640330; PubMed Central PMCID: PMC4313083.

39. Tuerdi A, Kinoshita M, Kamogashira T, Fujimoto C, Iwasaki S, Shimizu T, et al. Manganese superoxide dismutase influences the extent of noise-induced hearing loss in mice. Neurosci Lett. 2017;642:123-8. doi: 10.1016/j.neulet.2017.02.003. PubMed PMID: 28163078.

40. Pourbakht A, Yamasoba T. Ebselen attenuates cochlear damage caused by acoustic trauma. Hear Res. 2003;181(1-2):100-8. doi: 10.1016/s0378-5955(03)00178-3. PubMed PMID: 12855368.

41. Paciello F, Fetoni AR, Rolesi R, Wright MB, Grassi C, Troiani D, et al. Pioglitazone Represents an Effective Therapeutic Target in Preventing Oxidative/Inflammatory Cochlear Damage Induced by Noise Exposure. Frontiers in pharmacology. 2018;9:1103. doi: 10.3389/fphar.2018.01103. PubMed PMID: 30349478; PubMed Central PMCID: PMCPMC6187064.

42. Boero LE, Castagna VC, Di Guilmi MN, Goutman JD, Elgoyhen AB, Gomez-Casati ME. Enhancement of the Medial Olivocochlear System Prevents Hidden Hearing Loss. J Neurosci. 2018;38(34):7440-51. doi: 10.1523/JNEUROSCI.0363-18.2018. PubMed PMID: 30030403; PubMed Central PMCID: PMCPMC6104299. 43. Delmaghani S, Defourny J, Aghaie A, Beurg M, Dulon D, Thelen N, et al. Hypervulnerability to Sound Exposure through Impaired Adaptive Proliferation of Peroxisomes. Cell. 2015;163(4):894-906.

44. May LA, Kramarenko, II, Brandon CS, Voelkel-Johnson C, Roy S, Truong K, et al. Inner ear supporting cells protect hair cells by secreting HSP70. J Clin Invest. 2013;123(8):3577-87. doi: 10.1172/JCI68480. PubMed PMID: 23863716; PubMed Central PMCID: PMC3967657.

45. Puel JL, Ruel J, Gervais d'Aldin C, Pujol R. Excitotoxicity and repair of cochlear synapses after noisetrauma induced hearing loss. Neuroreport. 1998;9(9):2109-14. PubMed PMID: 9674603.

46. Puel JL, Saffiedine S, Gervais d'Aldin C, Eybalin M, Pujol R. Synaptic regeneration and functional recovery after excitotoxic injury in the guinea pig cochlea. C R Acad Sci III. 1995;318:67-75.

47. Pujol R, Lenoir M, Robertson D, Eybalin M, Johnstone BM. Kainic acid selectively alters auditory dendrites connected with cochlear inner hair cells. Hear Res. 1985;18:145-51.

48. Ruel J, Nouvian R, Gervais d'Aldin C, Pujol R, Eybalin M, Puel JL. Dopamine inhibition of auditory nerve activity in the adult mammalian cochlea. Eur J Neurosci. 2001;14(6):977-86. doi: 10.1046/j.0953816x.2001.01721.x. PubMed PMID: 11595036.

49. Yi E, Roux I, Glowatzki E. Dendritic HCN channels shape excitatory postsynaptic potentials at the inner hair cell afferent synapse in the mammalian cochlea. Journal of neurophysiology. 2010;103(5):2532-43. doi: 10.1152/jn.00506.2009. PubMed PMID: 20220080; PubMed Central PMCID: PMCPMC2867566. 50. Shrestha BR, Chia C, Wu L, Kujawa SG, Liberman MC, Goodrich LV. Sensory Neuron Diversity in the Inner Ear Is Shaped by Activity. Cell. 2018;174(5):1229-46 e17. doi: 10.1016/j.cell.2018.07.007. PubMed PMID: 30078709; PubMed Central PMCID: PMCPMC6150604. 
MYO7 SIRT3 DAPI
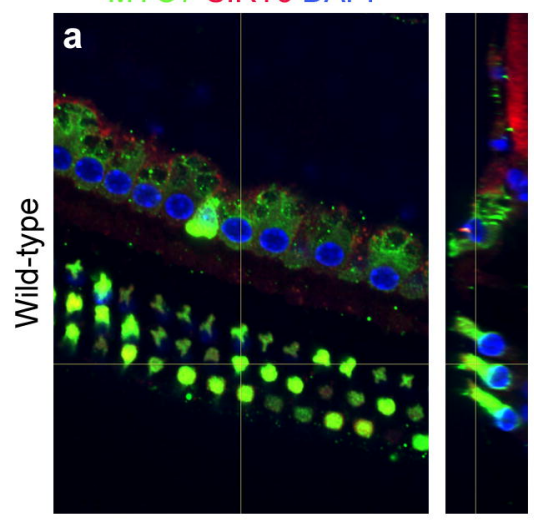

$a^{\prime}$

SIRT3
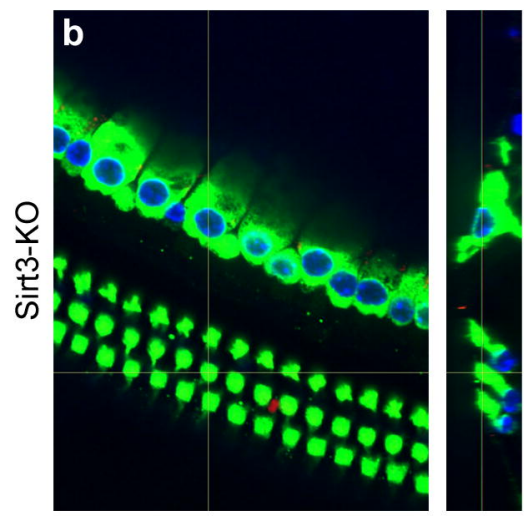

b'

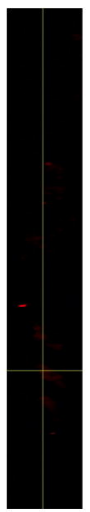



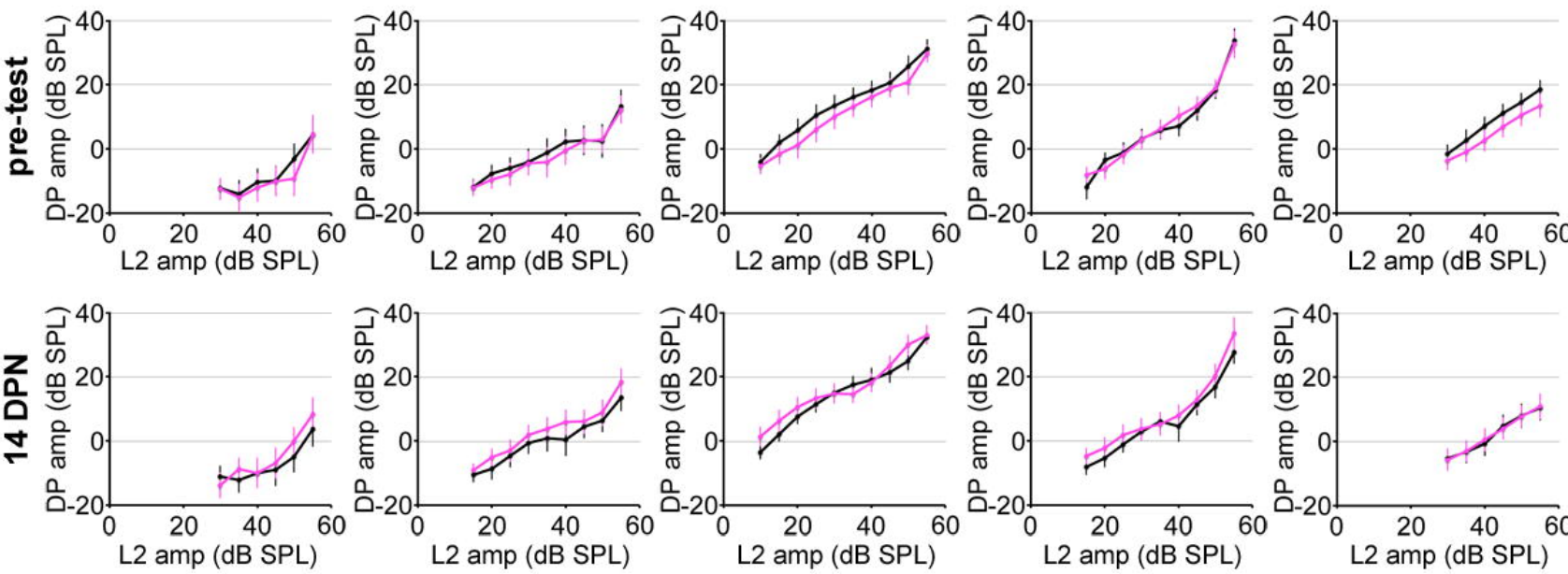
No Noise
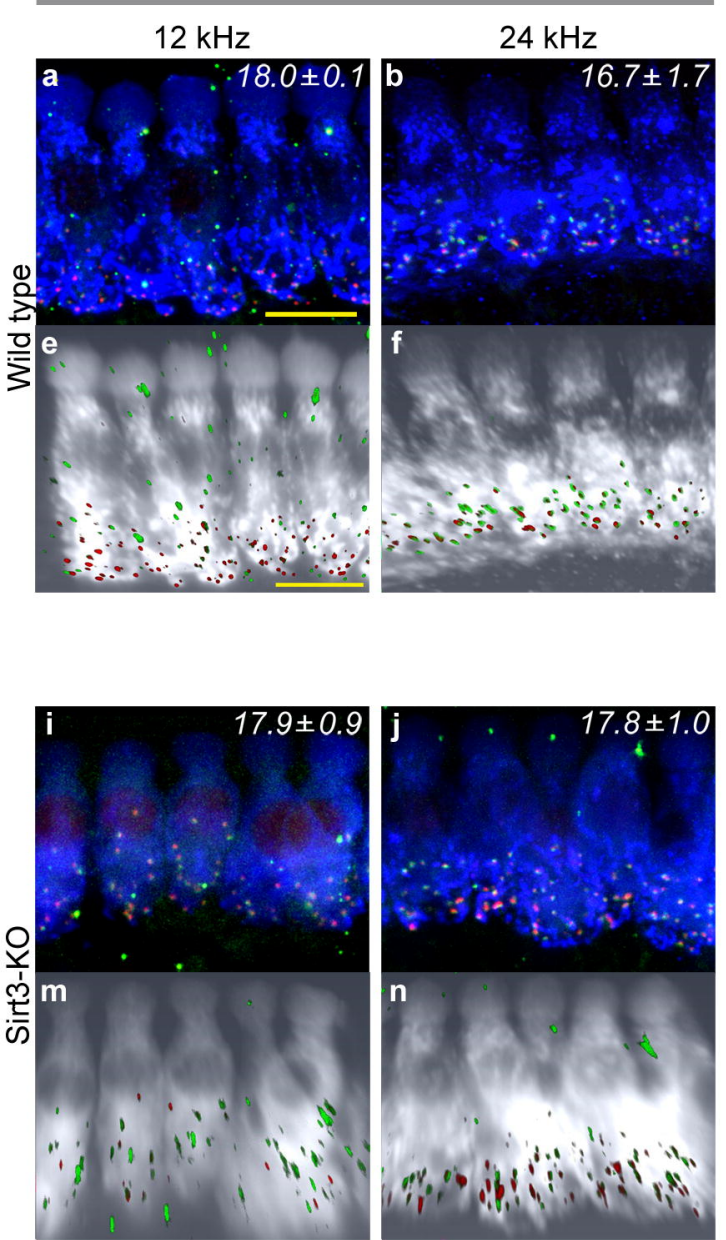

$24 \mathrm{kHz}$

$14.5 \pm 2.7$
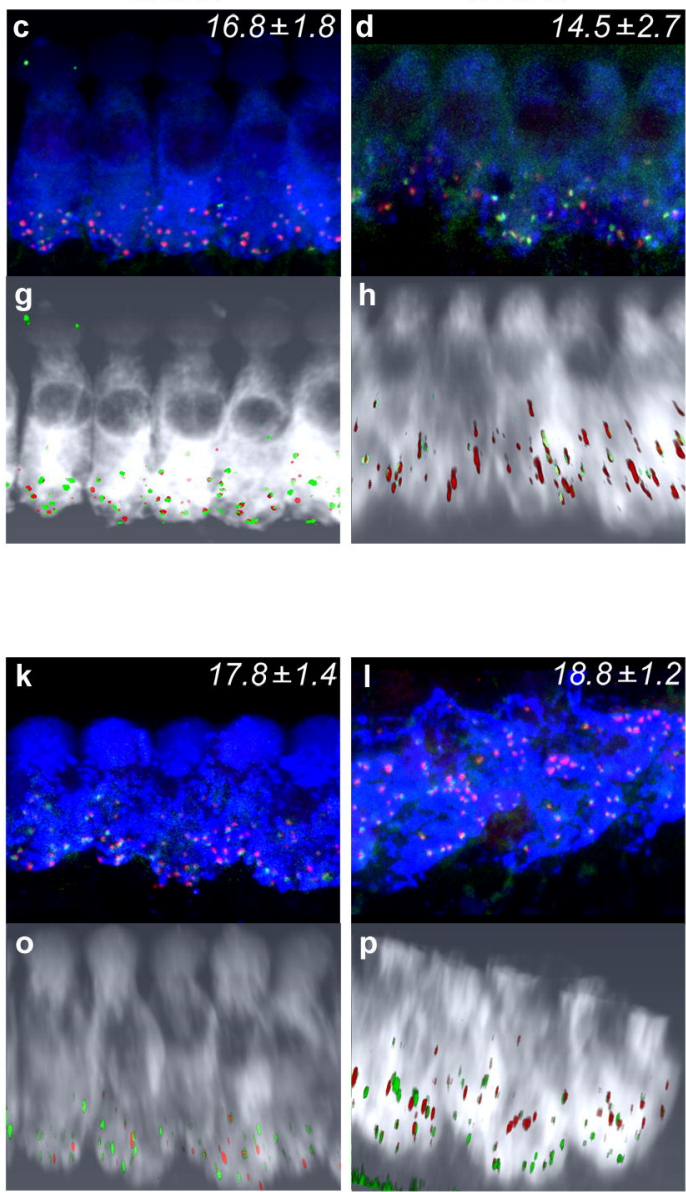